\title{
Compressed sensing real-time cine cardiovascular magnetic resonance: accurate assessment of left ventricular function in a single-breath-hold
}

Tomoyuki Kido ${ }^{1 *}$ D, Teruhito Kido ${ }^{1}$, Masashi Nakamura², Kouki Watanabe ${ }^{3}$, Michaela Schmidt ${ }^{4}$, Christoph Forman ${ }^{4}$ and Teruhito Mochizuki ${ }^{1}$

\begin{abstract}
Background: Cardiovascular cine magnetic resonance (CMR) accelerated by compressed sensing (CS) is used to assess left ventricular (LV) function. However, it is difficult for prospective CS cine CMR to capture the complete end-diastolic phase, which can lead to underestimation of the end-diastolic volume (EDV), stroke volume (SV), and ejection fraction (EF), compared to retrospective standard cine CMR. This prospective study aimed to evaluate the diagnostic quality and accuracy of single-breath-hold full cardiac cycle CS cine CMR, acquired over two heart beats, to quantify LV volume in comparison to multi-breath-hold standard cine CMR.

Methods: Eighty-one participants underwent standard segmented breath-hold cine and CS real-time cine CMR examinations to obtain a stack of eight contiguous short-axis images with same high spatial $(1.7 \times 1$. $7 \mathrm{~mm}^{2}$ ) and temporal resolution (41 ms). Two radiologists independently performed qualitative analysis of image quality (score, 1 [i.e., "nondiagnostic"] to 5 [i.e., "excellent"]) and quantitative analysis of the LV volume measurements.

Results: The total examination time was $113 \pm 7 \mathrm{~s}$ for standard cine CMR and $24 \pm 4 \mathrm{~s}$ for CS cine CMR $(p<0.0001)$. The CS cine image quality was slightly lower than standard cine ( $4.8 \pm 0.5$ for standard vs. $4.4 \pm 0.5$ for CS; $p<0.0001$ ). However, all image quality scores for CS cine were above 4 (i.e., good). No significant differences existed between standard and CS cine MR for all quantitative LV measurements. The mean differences with $95 \%$ confidence interval (Cl), based on Bland-Altman analysis, were $1.3 \mathrm{~mL}(95 \% \mathrm{Cl},-14.6$ - 17.2) for LV end-diastolic volume, $0.2 \mathrm{~mL}$ (95 \% Cl, -9.8 to10.3) for LV end-systolic volume, $1.1 \mathrm{~mL}$ (95\% Cl, -10.5 to 12.7) for LV stroke volume, $1.0 \mathrm{~g}$ ( $95 \% \mathrm{Cl},-11.2$ to 13.3) for LV mass, and $0.4 \%(95 \% \mathrm{Cl},-4.8-5.6)$ for $\mathrm{LV}$ ejection fraction. The interobserver and intraobserver variability for CS cine MR ranged from $-4.8-1.6 \%$ and from $-7.3-9.3 \%$, respectively, with slopes of the regressions ranging $0.88-1.0$ and $0.86-1.03$, respectively.
\end{abstract}

Conclusions: Single-breath-hold full cardiac cycle CS real-time cine CMR could evaluate LV volume with excellent accuracy. It may replace multi-breath-hold standard cine CMR.

Keywords: Cardiac function, Cardiovascular magnetic resonance, Compressed sensing, Left ventricular ejection fraction, Real-time imaging

\footnotetext{
*Correspondence: tomozo0421@gmail.com

'Department of Radiology, Ehime University Graduate School of Medicine,

Shitsukawa, Toon, Ehime 791-0295, Japan

Full list of author information is available at the end of the article
} 


\section{Background}

Accurate and reproducible left ventricular (LV) volume assessment, in particular the ejection fraction $(\mathrm{EF})$, is important in the management of various cardiac diseases because it is one of the strongest predictors of outcome [1-4]. Owing to high spatial and temporal resolution, a retrospective electrocardiogram (ECG)-gated breath-hold cine cardiovascular magnetic resonance $(\mathrm{CMR})$ is generally the reference standard for assessing LV volume [5-8]. Standard cine CMR is well established; however, it requires multiple scans to cover the entire left ventricle for functional evaluation. Thus, this approach is prone to involve a prolonged CMR examination. In addition, it is difficult for critically ill patients to tolerate acquisitions involving multiple breath-holds and long examination times. To overcome this shortcoming of the cine CMR, various acceleration techniques have been developed [9-12]. The recent development of the compressed sensing (CS) technique with sparse sampling and iterative reconstruction promises to reduce drastically the acquisition time of CMR [13-16]. Accelerating cine CMR with the CS approach improves patient compliance, and enables a cine acquisition of the entire left ventricle in a single-breath-hold and eventually shorter examination time. Some studies have demonstrated the utility of CS cine CMR for evaluating LV function [17-20].

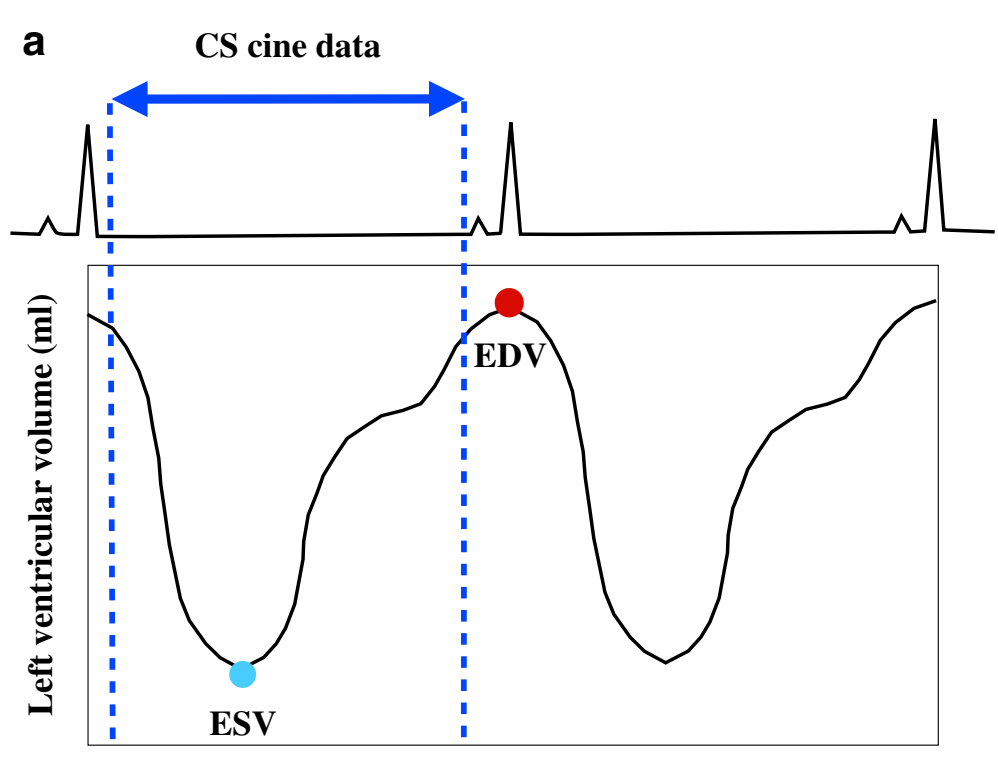

b CS cine data over two heart beats

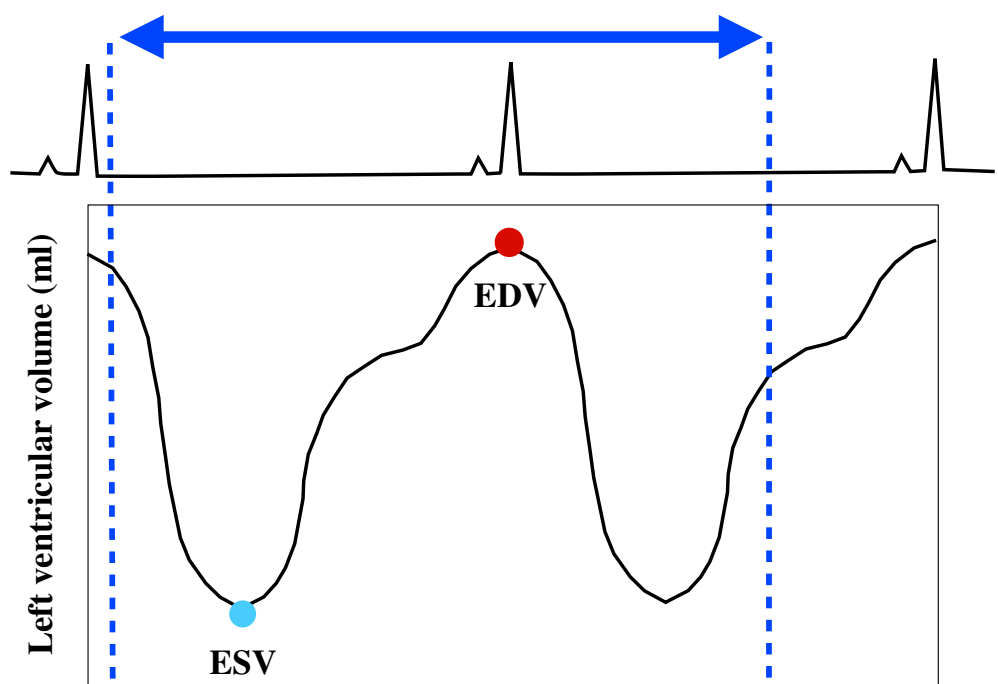

Fig. 1 Data acquisition of compressed sensing cine CMR for LV volume measurements. CS, compressed sensing; EDV, end-diastolic volume; ESV, end-systolic volume 
However, it is difficult for prospective ECG-triggered CS cine CMR to capture the complete end-diastolic phase in one heartbeat because it requires a finite time to detect the next ECG trigger $[19,20]$ as illustrated in Fig. 1a. When assessing LV function, this problem often leads to the underestimation of the end-diastolic volume (EDV), and accordingly the stroke volume (SV) and EF, compared to retrospective ECG-gated standard cine CMR [19]. To overcome this problem, we acquired the full cardiac cycle CS cine MR data over two heartbeats to capture the complete end-diastolic phase, which exists between the first and second heartbeat (Fig. 1b). The aim of this study was to evaluate the diagnostic quality and accuracy of single-breath-hold full cardiac cycle CS cine CMR for LV volume assessment in comparison to multi-breath-hold standard cine CMR.

\section{Methods}

\section{Study population}

This prospective study enrolled consecutive clinical patients with different cardiac conditions and healthy volunteers with no known cardiac disease. From August 2014 through May 2015, all participants underwent cine MR examinations, which included CS cine CMR and standard cine CMR sequences. All patients were clinically scheduled for CMR, and CS cine MR was part of the standard CMR protocol, as was standard cine CMR, which served as the standard of reference. The exclusion criteria for patient recruitment were arrhythmia and severely impaired breath-hold capacity.

\section{Cine magnetic resonance protocol}

All CMR examinations were performed using a clinical 3 T MR scanner (MAGNETOM Skyra; Siemens Healthcare, Erlangen, Germany). Scout images were obtained to plan the cardiac axis views. The segmented balanced steady-state free-precession sequence was used for the retrospective ECG-gated standard cine CMR scans of all participants. The short-axis cine CMR images were obtained in a stack of eight contiguous slices spanning the entire left ventricle from the base to the apex. The prospective ECG-triggered CS real-time cine CMR scans
Table 1 Imaging parameters

\begin{tabular}{lll}
\hline & Standard cine & CS cine \\
\hline ECG gating & Retrospective & Prospective \\
TE/TR (ms) & $1.4 / 3.2$ & $1.4 / 3.2$ \\
FOV (mm) & $350 \times 350$ & $350 \times 350$ \\
Image matrix & $208 \times 166$ & $208 \times 166$ \\
Spatial resolution (mm) & $1.7 \times 1.7$ & $1.7 \times 1.7$ \\
Temporal resolution (ms) & 41 & 41 \\
Slice thickness (mm) & 6 & 6 \\
Flip angle & 50 & 50 \\
Bandwidth (Hz/pixel) & 1145 & 960 \\
Cardiac phases & 25 & $19-31$ \\
Breath-holds ( $n$ ) & 4 & 1 \\
Acceleration factor & 3 & 80 \\
Iterative reconstruction & - & 12.8
\end{tabular}

CS compressed sensing, ECG electrocardiogram, FOV field of view, TE echo time, $T R$ repetition time

using a prototype sequence were performed immediately after the standard cine CMR scans. All scans were performed at end-inspiratory. The temporal resolution, spatial resolution, and slice orientations were identical in the two cine protocols. Detailed imaging parameters are listed in Table 1. The scan time for full cardiac cycle CS cine CMR was three heartbeats per slice. While one heartbeat was used to obtain the magnetization steady state, the remaining two heartbeats were utilized for data acquisition covering the full cardiac cycle CS cine data.

\section{Data acquisition and image reconstruction of the CS real-time cine}

Data acquisition is performed using sparse, incoherent sampling of $\mathrm{k}$-space. This is realized with a random distribution of the readouts on the Cartesian grid in k-space as illustrated in Fig. 2. Two parameters are used to adapt the sampling pattern, which are set to 7 and 13. While the first parameter defines the subsampling rate at $\mathrm{k}$-space center, it increases towards the high frequencies to a sub-sampling rate defined in

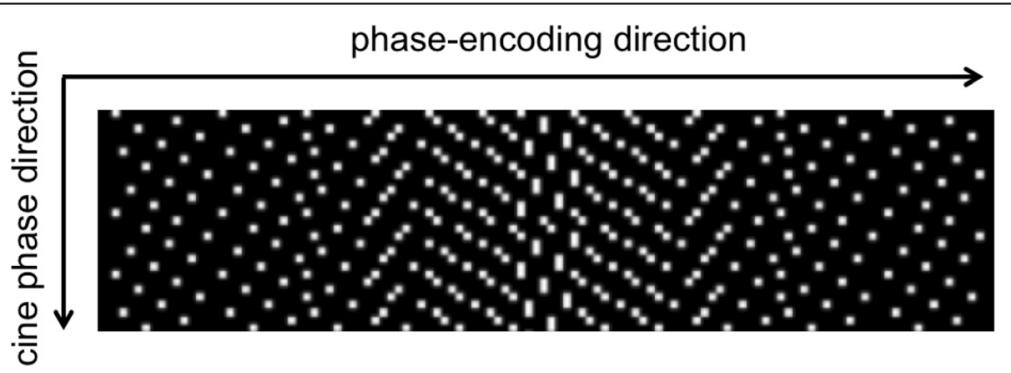

Fig. 2 Sampling pattern of compressed sensing cine CMR 
the second parameter. From frame-to-frame a random offset is applied which results in an incoherent temporal jitter.

Image reconstruction was performed with a nonlinear, iterative SENSE-type approach implementing spatio-temporal regularization using redundant Haar wavelets as described in [21]. The corresponding cost function was solved with a Fast Iterative ShrinkageThreshold Algorithm (FISTA) type optimization consisting of a gradient descend step for the quadratic terms and the evaluation of the proximal operator. The proximal operator is weighted with the regularization parameter, which was set to 0.001 and
0.005 for spatial and temporal regularization, respectively. The optimization was terminated after 40 iterations.

\section{Qualitative image quality analysis}

Two radiologists with 7 years and 5 years of experience in cardiac imaging assessed all short-axis cine CMR images independently with focus on the clearness of myocardial border and artifact. The image quality of each cine CMR image was evaluated visually and scored on a five-point scale: 1 = nondiagnostic quality, extensive artifact affecting volumetric analysis, $2=$ poor quality, moderate artifact affecting

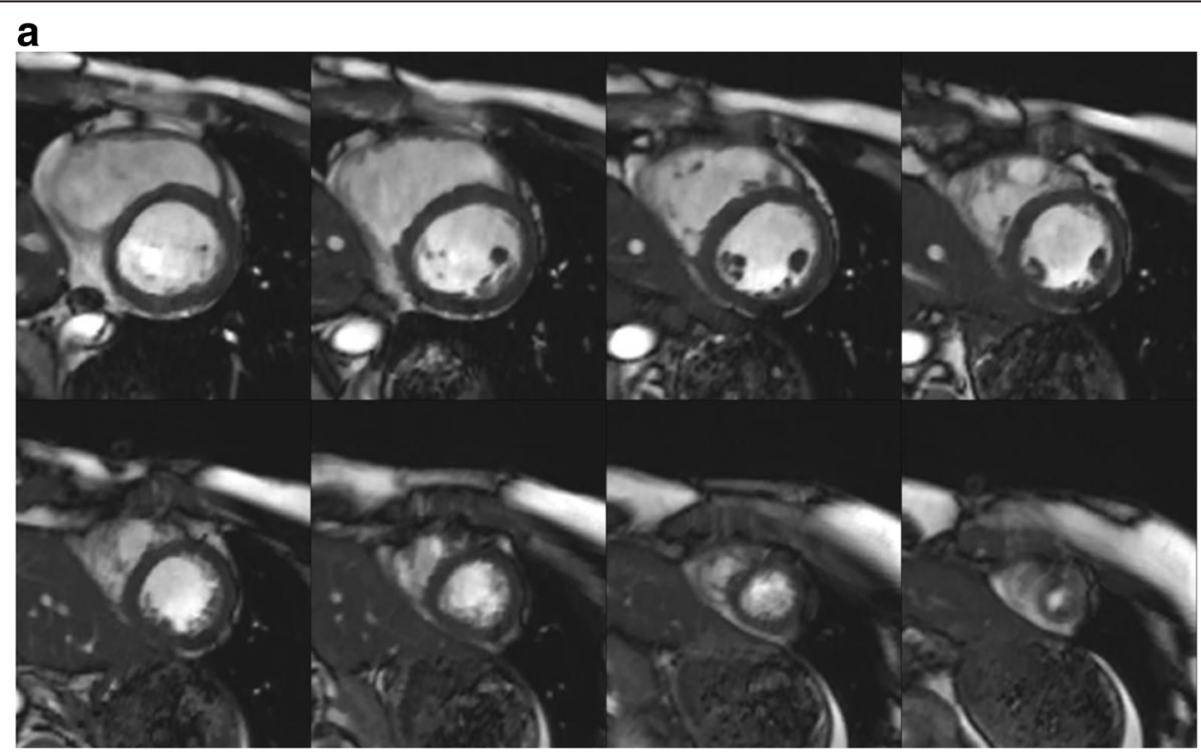

b

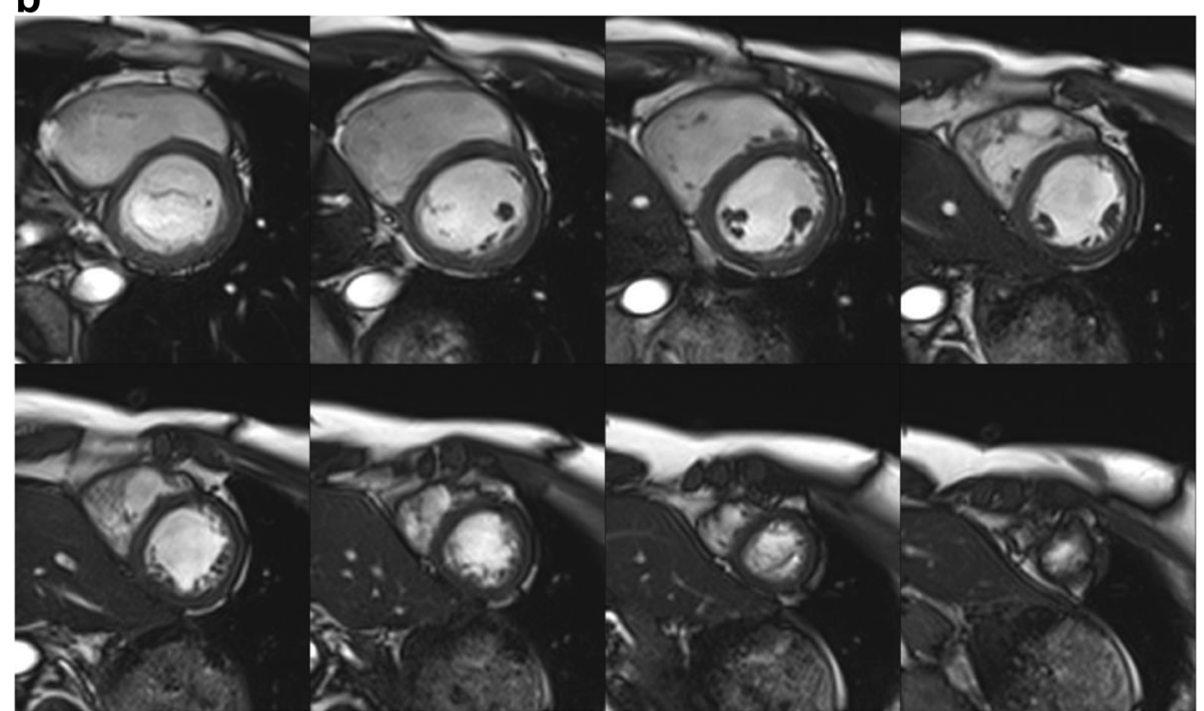

Fig. 3 Images acquired using compressed sensing cine and standard cine CMR. End-diastolic short-axis views of the left ventricle a by compressed sensing cine CMR and $\mathbf{b}$ by standard cine CMR. Both image sets were acquired from a 29-year-old healthy male volunteer. Both observers rated the image quality as excellent (i.e., score 5) for both images 
volumetric analysis, $3=$ adequate quality, mild artifact affecting volumetric analysis, $4=$ good quality, minimal not artifact affecting volumetric analysis, $5=$ excellent quality, no artifact.

\section{Quantitative LV volume analysis}

For quantitative measurements, the stack of eight contiguous short-axis slices of both cine CMR images was assessed independently by the two radiologists using the dedicated software package SYNAPSE VINCENT (Fujifilm Corp., Ltd, Tokyo, Japan). The epicardial and endocardial contours were automatically traced on the short-axis images. Contours rendered by automated analysis were reviewed and manually corrected, as necessary. The endocardial trabeculations and papillary muscles of the left ventricle were included in the LV cavity volumes [22-24]. The most basal slice with at least a semicircular muscular ring at the end-systolic phase was regarded as the base, and the most apical slice with a visible cavity at end-diastolic phase was regarded as the apex [25]. The LV volume and LV mass were calculated using the Simpson method. The end-systolic and end-diastolic phases were detected automatically by software, based on the smallest and largest LV volumes over the entire cardiac cycle.

\section{Statistical analysis}

The continuous data are expressed as the mean \pm the standard deviation (SD) or as the median (first quartile, third quartile), as appropriate, based on distribution. The Wilcoxon matched-pairs signed-rank test was used to compare image quality between standard cine CMR and CS cine CMR. The interobserver agreement on image quality was determined using the kappa test. The paired $t$ test was used to compare the scan time. The results of EDV, end-systolic volume (ESV), SV, LV mass, and EF on standard cine CMR and CS cine CMR were compared with the Wilcoxon matched-pairs signed-rank test. Linear regression and Bland-Altman analysis were used to evaluate the correlation and agreement between these LV measurements. In addition, interobserver and intraobserver variabilities in CS cine CMR were also determined by the same analysis. A $p$ value of less than 0.05 was considered statistically significant. All statistical analyses were performed by commercially available software (JMP version 11; SAS Institute, Cary, NC, USA). Sample size calculation was based on the primary outcome of the difference between the EF measures obtained from the two cine methods. Seventy-four participants were calculated to provide $80 \%$ power to detect more than $5 \%$ absolute difference in EF measures with a two-sided significance level of 0.05 , assuming a common SD for the mean EF measurement of $15 \%$. The EF margin was considered the clinically acceptable
Table 2 Characteristics of the study population

\begin{tabular}{lll}
\hline & Patients & Volunteers \\
\hline Number & 65 & 16 \\
Age (y) & $70.9 \pm 9.2$ & $28.1 \pm 4.4$ \\
Sex (female/male) & $18 / 47$ & $4 / 12$ \\
Height (m) & $160.8 \pm 9.5$ & $166.9 \pm 8.7$ \\
Weight (kg) & $62.1 \pm 13.2$ & $60.6 \pm 10.7$ \\
BMl (kg/m $\left.{ }^{2}\right)$ & $23.8 \pm 3.4$ & $21.6 \pm 2.2$ \\
HR (beats/min) & $62.2 \pm 10.3$ & $62.1 \pm 8.6$ \\
Cardiovascular risk factor & & - \\
$\quad$ Hypertension & $36(55 \%)$ & - \\
Dyslipidemia & $34(52 \%)$ & - \\
Diabetes mellitus & $26(40 \%)$ & - \\
Smoking & $16(25 \%)$ & - \\
Family history of CAD & $9(14 \%)$ & - \\
Cardiovascular disease & & - \\
CAD & $44(68 \%)$ & - \\
Cardiomyopathy & $14(22 \%)$ & - \\
Valve disease & $3(5 \%)$ & - \\
Other & $4(6 \%)$ & - \\
LVEF <50 \% & $20(31 \%)$ & - \\
\hline
\end{tabular}

The data are presented as the mean \pm standard deviation or as the median (first quartile, third quartile) or as the number (\%) of subjects

$B M I$ body mass index, $C A D$ coronary artery disease, $H R$ heart rate, $L V$ left ventricular, LVEDV left ventricular end diastolic volume, LVEF left ventricular ejection fraction, LVESV left ventricular end systolic volume, LVSV left ventricular stroke volume

range, based on previous research [26-30]. We ultimately enrolled 90 participants with the expectation of $18 \%$ attrition.

\section{Results}

Among the 90 enrolled participants, six patients with arrhythmia and three patients with severely impaired breath-hold capacity were excluded from the study. Eighty-one participants (65 patients and 16 volunteers) were ultimately used for qualitative analysis of image quality and for quantitative analysis of LV volume

Table 3 The LV volume measurements between standard cine and CS cine

\begin{tabular}{llll}
\hline & Standard cine $(n=81)$ & CS cine $(n=81)$ & $p$ \\
\hline LVEDV $(\mathrm{mL})$ & $121.0(105.8,161.7)$ & $122.2(103,159)$ & 0.28 \\
LVESV $(\mathrm{mL})$ & $48.3(34.8,79.1)$ & $50.9(32.9,77.8)$ & 0.77 \\
LVSV $(\mathrm{mL})$ & $73.5(63.8,85.2)$ & $73.4(63,83.5)$ & 0.15 \\
LV mass $(\mathrm{g})$ & $82.3(64.7,101.3)$ & $80.4(62.1,99.0)$ & 0.15 \\
LVEF (\%) & $61.3(50.5,68.0)$ & $58.8(50.7,67.5)$ & 0.10
\end{tabular}

The data are presented as the median (first quartile, third quartile) $C S$, compressed sensing; $L V$, left ventricular; $L V E D V$, left ventricular end diastolic volume; $L V E F$, left ventricular ejection fraction; $L V E S V$, left ventricular end systolic volume; LVSV, left ventricular stroke volume 
measurements. All 81 participants had a regular sinus rhythm with the mean heart rate of $62 \pm 10 \mathrm{bpm}$ (range, 42-88 bpm) during both cine CMR scans. The total examination time was $113 \pm 7 \mathrm{~s}$ (range, 100-130 s) for standard cine MR and 24 \pm 4 s (range, 16-34 s) for CS cine MR $(p<0.0001)$.

\section{Image quality}

Figure 3 shows representative sets of standard cine CMR and CS cine CMR images in eight short-axis slices from one healthy volunteer. Both cine CMR images showed excellent diagnostic image quality. The CS real-time cine CMR yielded slightly worse image quality scores than standard cine CMR $(4.8 \pm 0.5$ for standard vs. $4.4 \pm 0.5$ for CS; $p<0.0001)$. There was good interobserver agreement of image quality for standard cine CMR (kappa score $=0.82$ ) and for CS cine CMR (kappa score $=0.80)$.

\section{Left ventricular function}

All 81 standard and CS cine CMR images showed good to excellent image quality and they were sufficient to undergo quantitative analysis of the LV volume (Table 2). Multi-breath-hold standard cine CMR images were used as the standard reference for LV volume measurements (i.e., LVEF, LVEDV, LVESV, LVSV, and LV mass). Table 3 shows the median (first quartile, third quartile) values for the volumetric analysis of LV volume measurements and the analysis of the respective differences between standard and CS cine MR. There were no significant

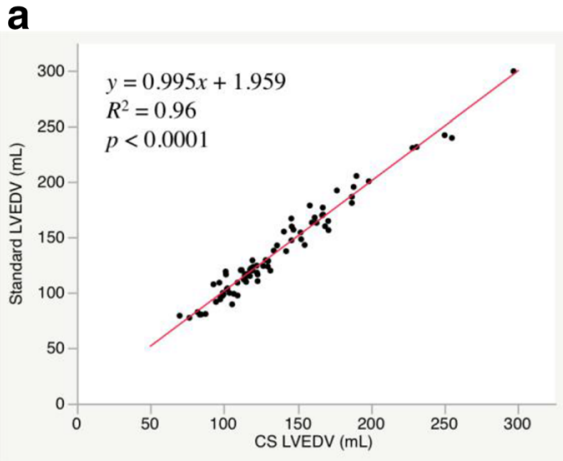

\section{b}
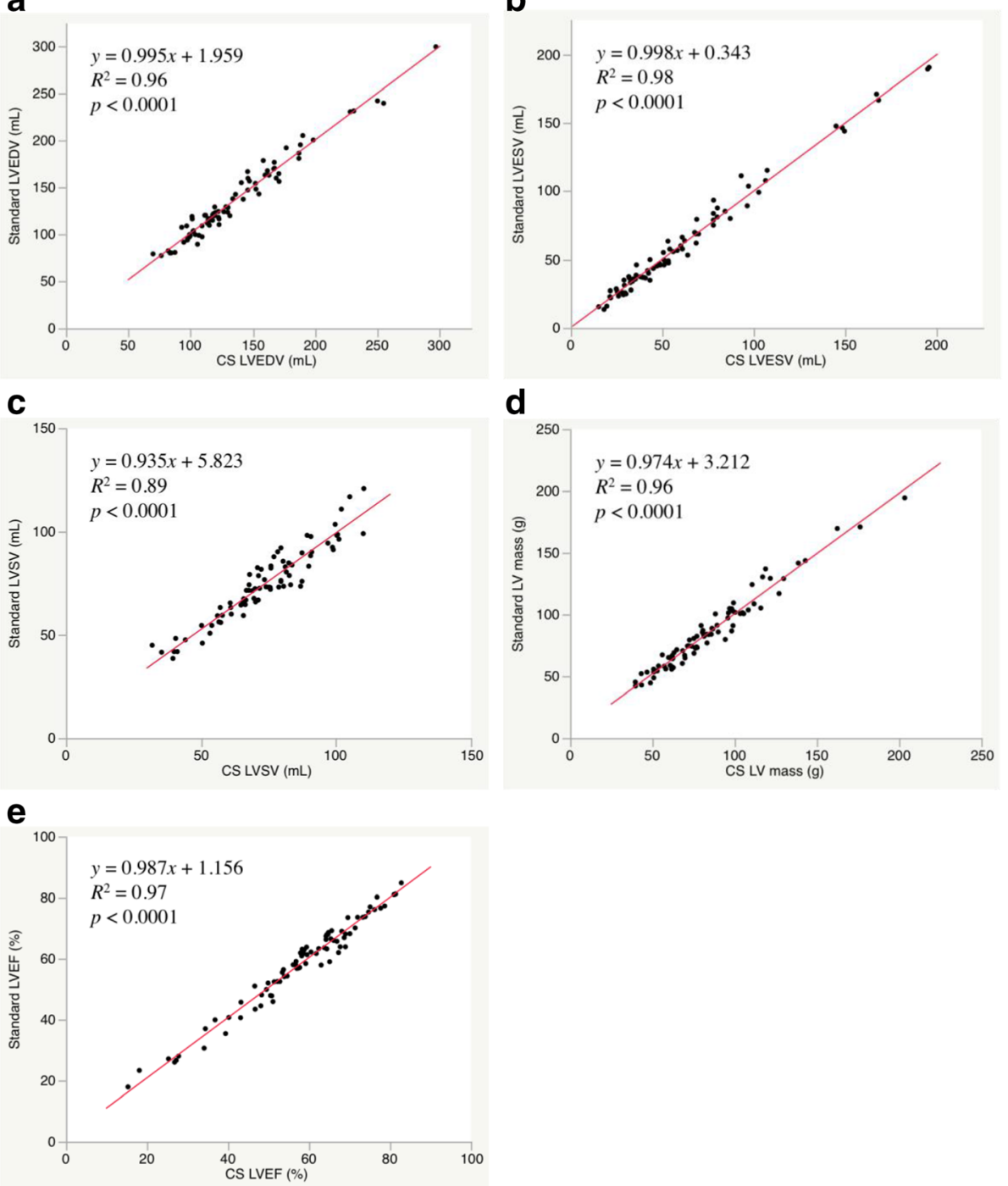

Fig. 4 Scatter plots for LV volume measurements by standard cine and CS cine. CS, compressed sensing; EF, ejection fraction; $L V$, left ventricular; LVEDV, left-ventricular end-diastolic volume; LVEF, left ventricular ejection fraction; LVESV, left ventricular end-systolic volume; LVSV, left-ventricular stroke volume; SD, standard deviation 
differences between standard and CS cine CMR for all LV volume measurements. The linear regression yielded good agreement between standard and CS cine CMR for all measurements (Fig. 4). Bland-Altman analysis revealed that the mean difference with $95 \%$ confidence interval (CI) between the standard cine CMR and the CS cine CMR were $1.3 \mathrm{~mL}$ (95\% CI, $-14.6 \mathrm{~mL}$ to $17.2 \mathrm{~mL}$ ) for LVEDV, $0.2 \mathrm{~mL}$ (95\% CI, $-9.8 \mathrm{~mL}$ to $10.3 \mathrm{~mL}$ ) for LVESV, $1.1 \mathrm{~mL}(95 \% \mathrm{CI},-10.5 \mathrm{~mL}$ to $12.7 \mathrm{~mL}$ ) for LVSV, $1.0 \mathrm{~g}$ (95\% CI, $-11.2 \mathrm{~g}$ to $13.3 \mathrm{~g}$ ) for LV mass, and $0.4 \%$ (95 \% CI, $-4.8-5.6 \%)$ for LVEF (Fig. 5). The interobserver and intraobserver variability for CS cine
CMR ranged from $-4.8-1.6 \%$ and from $-7.3-9.3 \%$, respectively, with the slopes of regression ranging $0.88-1.0$ and $0.86-1.03$, respectively (Table 4 ).

\section{Discussion}

In this prospective study, single-breath-hold full cardiac cycle CS cine CMR showed high agreement for the volumetric analysis of the left ventricle, compared to the current reference standard multi-breath-hold cine CMR. Some previous studies have also shown that CS cine CMR is similar to standard cine CMR in image quality, and that the LV volume measurements were in good
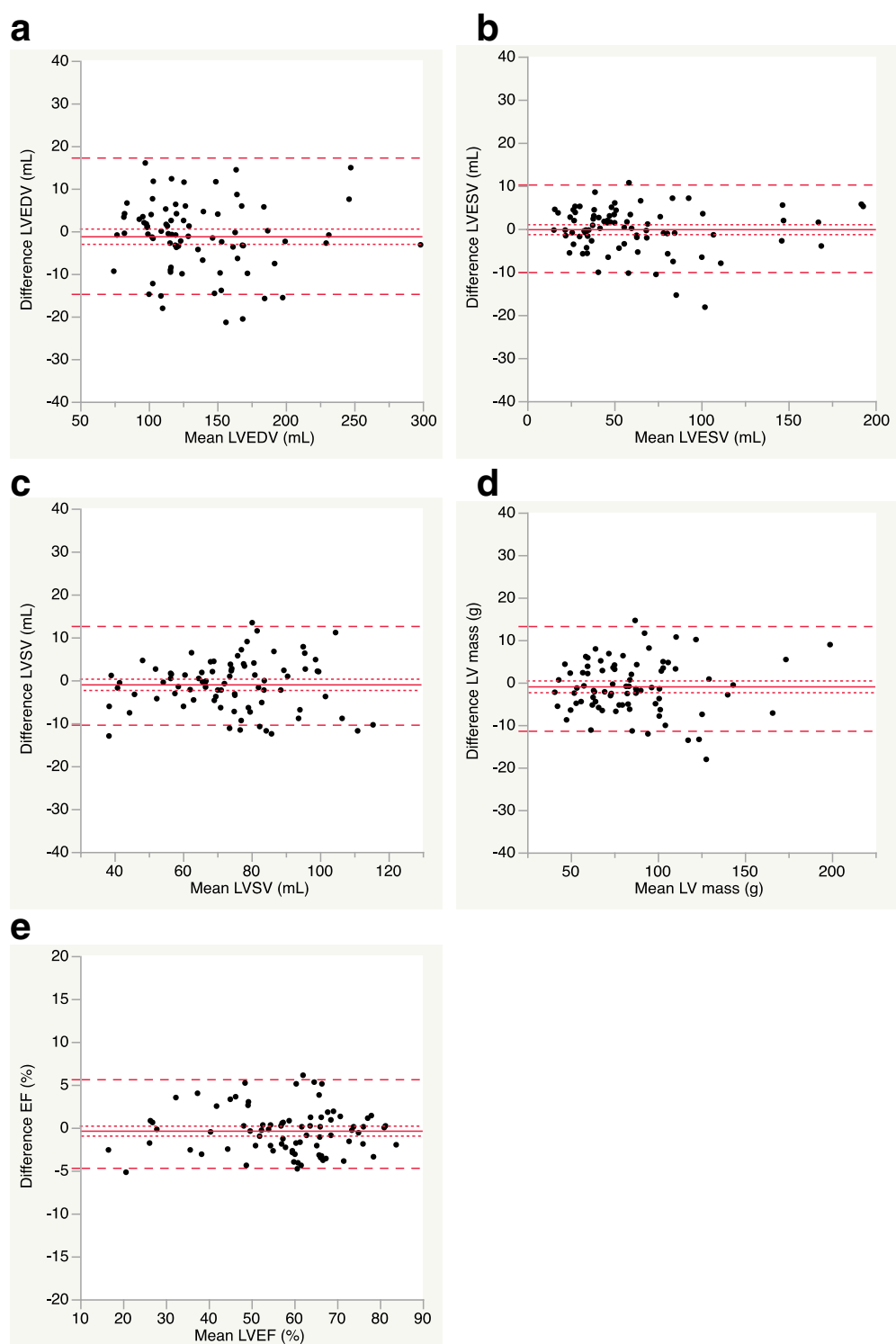

Fig. 5 Bland-Altman plots for LV volume measurements by standard cine and CS cine. The solid line indicates the difference between two sequences; the long dashed lines indicate the $95 \%$ limits of agreement interval (i.e., the mean \pm 1.96 SD); and the short dashed lines indicate the $95 \%$ confidence interval of the mean difference CS, compressed sensing; $E F$, ejection fraction; $L V$, left ventricular; $L V E D V$, left-ventricular end-diastolic volume; LVEF, left-ventricular ejection fraction; LVESV, left-ventricular end-systolic volume; LVSV, left-ventricular stroke volume; SD, standard deviation 
Table 4 Intraobserver and interobserver variability of the CS cine CMR

\begin{tabular}{|c|c|c|c|c|c|}
\hline & $\begin{array}{l}\text { Difference } \\
\text { (mean } \pm S D \text { ) }\end{array}$ & $\begin{array}{l}\text { Variability [\%] } \\
\text { (mean } \pm \text { SD) }\end{array}$ & $R^{2}$ & Slope & $p$ \\
\hline \multicolumn{6}{|l|}{ Intraobserver } \\
\hline LVEDV (mL) & $0.3 \pm 11.6$ & $1.0 \pm 4.7$ & 0.98 & 1.0 & $<0.0001$ \\
\hline LVESV (mL) & $0.4 \pm 3.8$ & $1.1 \pm 7.0$ & 0.99 & 0.99 & $<0.0001$ \\
\hline LVSV (mL) & $0.2 \pm 11.1$ & $1.6 \pm 7.7$ & 0.91 & 0.92 & $<0.0001$ \\
\hline LV mass (g) & $-5.4 \pm 9.4$ & $-4.8 \pm 8.8$ & 0.95 & 0.88 & $<0.0001$ \\
\hline LVEF (\%) & $0.1 \pm 2.9$ & $0.7 \pm 4.9$ & 0.97 & 0.96 & $<0.0001$ \\
\hline \multicolumn{6}{|l|}{ Interobserver } \\
\hline LVEDV (mL) & $8.0 \pm 5.8$ & $6.4 \pm 5.1$ & 0.98 & 1.03 & $<0.0001$ \\
\hline LVESV (mL) & $1.7 \pm 4.8$ & $2.6 \pm 9.8$ & 0.99 & 1.03 & $<0.0001$ \\
\hline LVSV (mL) & $6.3 \pm 6.0$ & $9.3 \pm 8.9$ & 0.89 & 0.98 & $<0.0001$ \\
\hline LV mass (g) & $-6.3 \pm 11.5$ & $-7.3 \pm 13.2$ & 0.88 & 0.86 & $<0.0001$ \\
\hline LVEF (\%) & $1.5 \pm 3.4$ & $2.9 \pm 6.3$ & 0.95 & 0.97 & $<0.0001$ \\
\hline
\end{tabular}

Bland-Altman plots highlight the mean difference and the standard deviation of the difference between the two measurements. Variability [\%] is the absolute value of the difference between the two measurements divided by the mean of the two measurements

CS compressed sensing, LV left ventricular, LVEDV left ventricular end diastolic volume, LVEF left ventricular ejection fraction, LVESV left ventricular end systolic volume, LVSV left ventricular stroke volume, MR magnetic resonance, $S D$ standard deviation

agreement. However, other investigators also report that CS cine CMR with prospective ECG-triggering cannot detect the very first and last phases of the cardiac cycle $[19,20]$. This limitation often leads to the underestimation of EDV, SV, and EF. Our results suggested that single-breath-hold full cardiac cycle CS cine CMR could overcome this limitation by acquiring data over two heartbeats. In addition, an accurate assessment of LV function depends on spatial and temporal resolution [31]. Compromised spatial and temporal resolution often causes a substantial problem in image quality and in the accuracy of highly accelerated cine CMR in comparison to the standard cine CMR [32]. In our study, the high spatial $(1.7 \mathrm{~mm} \times 1.7 \mathrm{~mm})$ and temporal $(41 \mathrm{~ms})$ resolutions of CS cine CMR were identical to those of standard cine CMR and translated into good image quality and high agreement for all LV measurements. Furthermore, we found that the variability of the LVEF (95\% CI, $-4.8-5.6 \%$ ) between standard cine CMR and CS cine CMR measured in our study was comparable to the interstudy variability of LVEF measurements of standard cine CMR (95\% CI, $-4.1-4.3 \%)$ reported in previous research $[8,33]$. These results indicate that the current single-breath-hold CS cine CMR was accurate and sufficiently reproducible to replace multi-breath-hold standard cine CMR. In addition, a rapid CS cine CMR examination is more cost-effective than multi-breath-hold standard cine CMR and particularly beneficial for ill patients who cannot tolerate prolonged examination times.

The CS cine CMR yielded a slightly worse image quality score, compared to standard cine CMR. Some CS cine CMR images had worse image quality because foldover artifacts and flow-related artifacts occurred in the phase-encoding direction during the systolic phase. However, image quality scores were above 4 (i.e., good) for all CS cine CMR. This result suggested that acceptable diagnostic image quality could be achieved by CS cine CMR. Moreover, all patients with arrhythmia or impaired breath-hold capacity were excluded in this study because we were using the standard cine CMR images as the standard reference for LV measurements. In this study, this exclusion may have been advantageous in terms of image quality for standard cine CMR. These excluded patients often have image deterioration on the retrospective standard cine CMR image and impaired accuracy in the volumetric analysis in real clinical settings $[34,35]$. On the other hand, the prospective CS real-time cine CMR has the advantage of being inherently insensitive to arrhythmia or respiratory motion because of the single-shot acquisition [36, 37]. In this study, the maximal number of cine CMR slices that a single-breath-hold can support will be limited by the ability of breath-hold. A variety of acceleration techniques could develop free-breathing cine CMR to overcome this limitation [38, 39]. The CS real-time cine CMR is also suited to be scanned in freebreathing as shown in another study [40]. In the future, further evaluation is needed to assess the clinical utility of the current CS cine CMR in free-breathing or in patients with arrhythmia.

\section{Limitations}

The CS cine CMR is adequate for LV volume measurements; however, there are some limitations to this study. First, we did not evaluate regional myocardial wall motion abnormalities. This assessment is also important and a desired application of cardiac cine CMR in clinical practice. However, we could visually detect a regional myocardial wall motion abnormality of patients with myocardial infarction using the CS cine image and the standard cine image (Fig. 6). We expect that further examination will reveal the accuracy of CS cine CMR for assessing regional wall motion in patients with myocardial infarction. Second, the EF in this study group covered almost the entire range of clinical relevance (i.e., $18-85 \%$ ), but only $25 \%$ of patients showed an EF less than $50 \%$. Third, we didn't make an experimental study on effects of temporal and spatial resolution for CS reconstruction. We'll need further consideration about the effect of higher temporal resolution than $41 \mathrm{~ms}$ for CS reconstruction. 


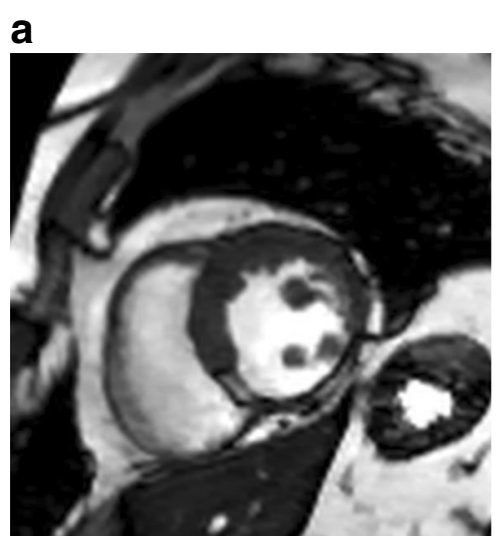

b

C

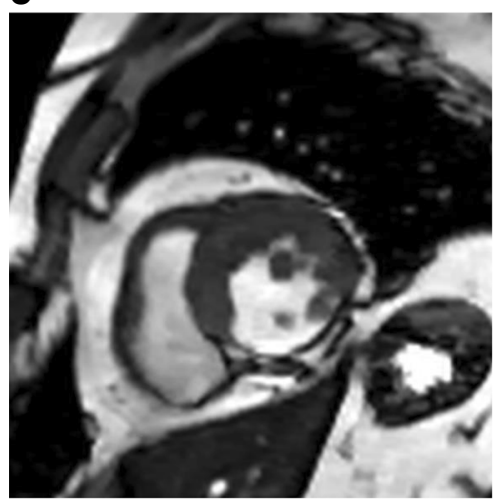

e

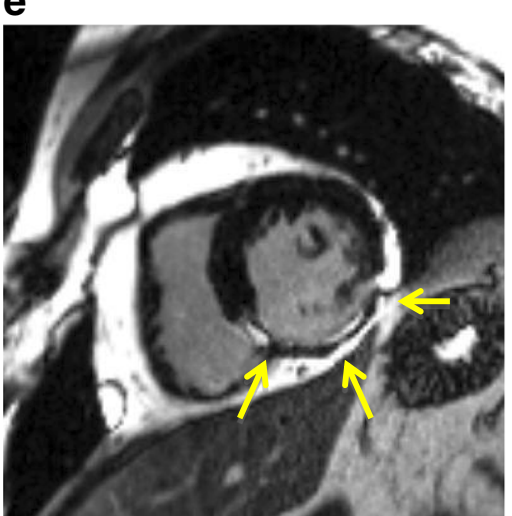

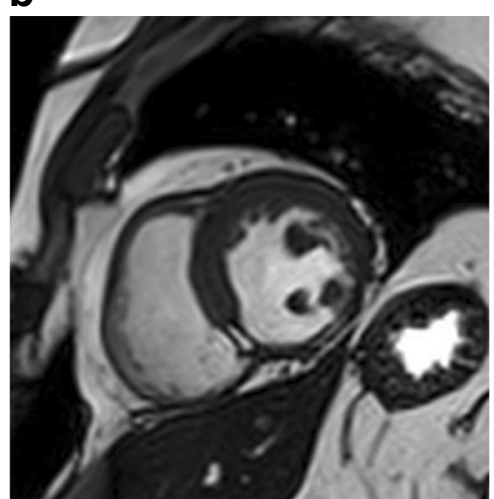

d

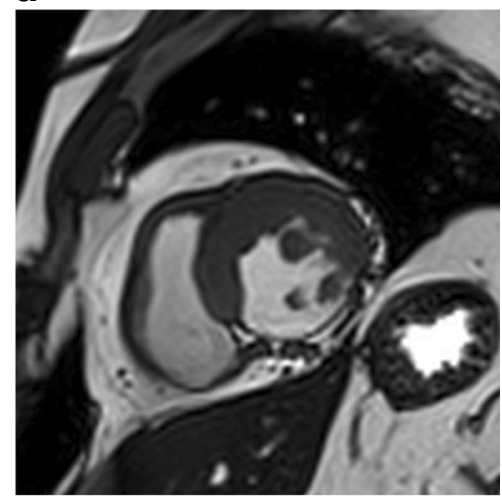
. 


\section{Abbreviations}

$\mathrm{Cl}$, confidence interval; CS, compressed sensing; EDV, end-diastolic volume; EF, ejection fraction; ESV, end-systolic volume; LV, left ventricular; CMR, cardiovascular magnetic resonance; SD, standard deviation; SV, stroke volume.

\section{Acknowledgements}

The authors are grateful to Yoshiaki Komori and Yuta Urushibata of Siemens Healthcare K.K. (Tokyo, Japan) for the optimization of the sequence parameters and image quality in the study.

\section{Funding}

Not applicable.

\section{Availability of supporting data and materials} Not applicable.

\section{Authors' contributions}

TK conceived the study, quantitatively measured cardiac function, analyzed image quality, performed statistical analysis, and drafted the manuscript. TK participated in design of the study, assisted in the interpretation of the results, and helped to revise the manuscript. MN quantitatively measured cardiac function and analyzed image quality and helped to revise the manuscript. KW participated in the design of the study and coordination and helped to revise the manuscript. MS contributed to the sequence development, implementation on the scanner and helped to revise the manuscript. CF contributed to the sequence development and implementation on the scanner and helped to revise the manuscript. TM assisted in the interpretation of the results and helped to revise the manuscript. All authors have read and approved the final manuscript.

\section{Authors' information}

Not applicable.

\section{Competing interests}

The authors declare that they have no competing interests.

\section{Consent for publication}

All participants in this study gave written consent to participate and to publish.

\section{Ethical approval and consent to participate}

The study protocol was approved by the Ethical Review Board in Saiseikai Matsuyama Hospital. All participants in this study gave written consent to participate and to publish.

\section{Author details}

'Department of Radiology, Ehime University Graduate School of Medicine, Shitsukawa, Toon, Ehime 791-0295, Japan. ${ }^{2}$ Department of Radiology, Saiseikai Matsuyama Hospital, 880-2, Yamanishi, Matsuyama, Ehime 791-8026, Japan. ${ }^{3}$ Department of Cardiology, Saiseikai Matsuyama Hospital, 880-2, Yamanishi, Matsuyama, Ehime 791-8026, Japan. ${ }^{4}$ Siemens Healthcare GmbH, Allee am Roethelheimpark 2, 91052 Erlangen, Germany.

Received: 30 June 2016 Accepted: 29 July 2016

Published online: 24 August 2016

\section{References}

1. Curtis JP, Sokol SI, Wang Y, Rathore SS, Ko DT, Jadbabale F, et al. The association of left ventricular ejection fraction, mortality, and cause of death in stable outpatients with heart failure. J Am Coll Cardiol. 2003;42:736-42.

2. Knauth AL, Gauvreau K, Powell AJ, Landzberg MJ, Walsh EP, Lock JE, et al. Ventricular size and function assessed by cardiac MRI predict major adverse clinical outcomes late after tetralogy of Fallot repair. Heart. 2008;94:211-6.

3. Bamberg F, Parhofer KG, Lochner E, Marcus RP, Theisen D, Findeisen HM, et al. Diabetes mellitus: long-term prognostic value of whole-body MR imaging for the occurrence of cardiac and cerebrovascular events. Radiology. 2013:269:730-7.

4. White HD, Norris RM, Brown MA, Brandt PW, Whitlock RM, Wild CJ. Left ventricular end-systolic volume as the major determinant of survival after recovery from myocardial infarction. Circulation. 1987;76:44-51.
5. Rathi VK, Biedermann RW. Imaging of ventricular function by cardiovascular magnetic resonance. Curr Cardiol Rep. 2004;6:55-61.

6. Alfakih K, Plein S, Thiele H, Jones T, Ridgway JP, Sivananthan MU. Normal human left and right ventricular dimensions for MRI as assessed by turbo gradient echo and steady-state free precession imaging sequences. J Magn Reson Imaging. 2003;17:323-9.

7. Moon JC, Lorenz CH, Francis JM, Smith GC, Pennell DJ. Breath-hold FLASH and FISP cardiovascular MR imaging: left ventricular volume differences and reproducibility. Radiology. 2002;223:789-97.

8. Grothues F, Smith GC, Moon JC, Bellenger NG, Collins P, Klein HU, et al. Comparison of interstudy reproducibility of cardiovascular magnetic resonance with two-dimensional echocardiography in normal subjects and in patients with heart failure or left ventricular hypertrophy. Am J Cardiol. 2002;90:29-34.

9. Kozerke S, Plein S. Accelerated CMR using zonal, parallel and prior knowledge driven imaging methods. J Cardiovasc Magn Reson. 2008;10:29.

10. Jahnke C, Nagel E, Gebker R, Bormstedt A, Schnackenburg B, Kozerke S, et al. Four-dimensional single-breath-hold magnetic resonance imaging using kt-BLAST enables reliable assessment of left- and right-ventricular volumes and mass. J Magn Reson Imaging. 2007;25:737-42.

11. Eberle HC, Nassenstein K, Jensen C, Schlosser T, Sabin GV, Naber CK, et al. Rapid MR assessment of left ventricular systolic function after acute myocardial infarction using single-breath-hold cine imaging with the temporal parallel acquisition technique (TPAT) and 4D guide-point modelling analysis of left ventricular function. Eur Radiol. 2010;20:73-80.

12. Schwitter J, Oelhafen M, Wyss BM, Kozerke S, Amann-Vesti B, Lüscher TF, et al. 2D-spatially-selective real-time magnetic resonance imaging for the assessment of microvascular function and its relation to the cardiovascular risk profile. J Cardiovasc Magn Reson. 2006;8:759-69.

13. Candes EJ, Wakin MB. An introduction to compressive sampling. IEEE Signal Process Mag. 2008;25:21-30.

14. Lustig M, Donoho D, Pauly JM. Sparse MRI: the application of compressed sensing for rapid MR imaging. Magn Reson Med. 2007;58:1182-95.

15. Gamper U, Boesiger P, Kozerke S. Compressed sensing in dynamic MRI. Magn Reson Med. 2008;59:365-73.

16. Jung H, Sung K, Nayak KS, Kim EY, Ye JC. k-t FOCUSS: a general compressed sensing framework for high resolution dynamic MRI. Magn Reson Med. 2009:61:103-16.

17. Wech T, Lemke A, Medway D, Stork LA, Lygate CA, Neubauer S, et al. Accelerating cine-MR imaging in mouse hearts using compressed sensing. J Magn Reson Imaging. 2011;34:1072-9.

18. Bassett EC, Kholmovski EG, Wilson BD, DiBella EV, Dosdall DJ, Ranjan R, et al. Evaluation of highly accelerated real-time cardiac cine MRI in tachycardia. NMR Biomed. 2014:27:175-82.

19. Vincenti G, Monney P, Chaptinel J, Rutz T, Coppo S, Zenge MO, et al. Compressed sensing single-breath-hold CMR for fast quantification of LV function, volumes, and mass. JACC Cardiovasc Imaging. 2014;7:882-92.

20. Feng L, Srichai MB, Lim RP, Harrison A, King W, Adluru G, et al. Highly accelerated real-time cardiac cine MRI using k-t SPARSE-SENSE. Magn Reson Med. 2013;70:64-74.

21. Liu J, Rapin J, Chang T, Lefebvre A, Zenge M, Mueller E, et al. Dynamic cardiac MRI reconstruction with weighted redundant Haar wavelets. ISMRM. 2012;20:178.

22. Miller S, Simonetti OP, Carr J, Carr J, Kramer U, Finn JP. MR imaging of the heart with cine true fast imaging with steady-state precession: influence of spatial and temporal resolutions on left ventricular functional parameters. Radiology. 2002;223:263-9.

23. van Geuns RJ, Baks T, Gronenschild EH, Aben JP, Wielopolski PA, Cademartiri $F$, et al. Automatic quantitative left ventricular analysis of cine MR images by using three-dimensional information for contour detection. Radiology. 2006; 240:215-21.

24. Sievers B, Kirchberg S, Bakan A, Franken U, Trappe HJ. Impact of papillary muscles in ventricular volume and ejection fraction assessment by cardiovascular magnetic resonance. J Cardiovasc Magn Reson. 2004; 6:9-16.

25. Wu Y, Tadamura E, Yamamuro M, Kanao S, Okayama S, Ozasa N, et al. Estimation of global and regional cardiac function using 64-slice computed tomography: a comparison study with echocardiography, gated-SPECT and cardiovascular magnetic resonance. Int J Cardiol. 2008;128:69-76.

26. Pattynama PM, Lamb HJ, Van der Velde EA, van der Wall EE, de Roos A. Left ventricular measurements with cine and spin echo MR imaging: 
a study of reproducibility with variance component analysis. Radiology. 1993;187:261-8.

27. Cohn PF, Levine JA, Bergeron GA, Gorlin R. Reproducibility of the angiographic left ventricular ejection fraction in patients with coronary artery disease. Am Heart J. 1974;88:713-20.

28. Gordon EP, Schnittger I, Fitzgerald PJ, Williams P, Popp RL. Reproducibility of left ventricular volumes by two-dimensional echocardiography. J Am Coll Cardiol. 1983;2:506-13.

29. Dorosz JL, Lezotte DC, Weitzenkamp DA, Allen LA, Salcedo EE. Performance of 3-dimensional echocardiography in measuring left ventricular volumes and ejection fraction: a systematic review and meta-analysis. J Am Coll Cardiol. 2012;59:1799-808.

30. Malm S, Frigstad S, Sagberg E, Larsson H, Skjaerpe T. Accurate and reproducible measurement of left ventricular volume and ejection fraction by contrast echocardiography: a comparison with magnetic resonance imaging. J Am Coll Cardiol. 2004;44:1030-5.

31. Setser RM, Fischer SE, Lorenz CH. Quantification of left ventricular function with magnetic resonance images acquired in real time. J Magn Reson Imaging. 2000;12:430-8.

32. Muthurangu V, Lurz P, Critchely JD, Deanfield JE, Taylor AM, Hansen MS. Real-time assessment of right and left ventricular volumes and function in patients with congenital heart disease by using high spatiotemporal resolution radial k-t SENSE1. Radiology. 2008;248:782-91.

33. Danilouchkine M, Westenberg J, De Roos A, Reiber J, Lelieveldt P. Operato induced variability in cardiovascular MR: left ventricular measurements and their reproducibility. J Cardiovasc Magn Reson. 2005;7:447-57.

34. Klinke V, Muzzarelli S, Lauriers N, Locca D, Vincenti G, Monney P, et al. Quality assessment of cardiovascular magnetic resonance in the setting of the European CMR Registry: description and validation of standardized criteria. J Cardiovasc Magn Reson. 2013;15:55.

35. Marks B, Mitchell DG, Simelaro JP. Breath-holding in healthy and pulmonary-compromised populations: effects of hyperventilation and oxygen inspiration. J Magn Reson Imaging. 1997;7:595-7.

36. Voit D, Zhang S, Unterberg-Buchwald C, Sohns JM, Lotz J, Frahm J. Real-time cardiovascular magnetic resonance at $1.5 \mathrm{~T}$ using balanced SSFP and 40 ms resolution. Cardiovasc Magn Reson. 2013;15:79.

37. Aandal G, Nadig V, Yeh V, Rajiah P, Jenkins T, Sattar A, et al. Evaluation of left ventricular ejection fraction using through-time radial GRAPPA. J Cardiovasc Magn Reson. 2014;16:79.

38. Xue H, Kellman P, Larocca G, Arai AE, Hansen MS. High spatial and temporal resolution retrospective cine cardiovascular magnetic resonance from shortened free breathing real-time acquisitions. J Cardiovasc Magn Reson. 2013;15:102.

39. Sayin O, Saybasili H, Zviman MM, Griswold M, Halperin H, Seiberlich N, et al. Real-time free-breathing cardiac imaging with self-calibrated through-time radial GRAPPA. Magn Reson Med 2016; doi:10.1002/mrm.26112.

40. Goebel J, Nensa F, Bomas B, Schemuth HP, Maderwald S, Gratz M, et al. Real-time SPARSE-SENSE cardiac cine MR imaging: optimization of image reconstruction and sequence validation. Eur Radiol. 2016. doi:10.1007/ s00330-016-4301-y. Epub ahead of print.

41. Saybasili H, Herzka DA, Seiberlich N, Griswold MA. Real-time imaging with radial GRAPPA: implementation on a heterogeneous architecture for low-latency reconstructions. Magn Reson Imaging. 2014;32:747-58.

\section{Submit your next manuscript to BioMed Central and we will help you at every step:}

- We accept pre-submission inquiries

- Our selector tool helps you to find the most relevant journal

- We provide round the clock customer support

- Convenient online submission

- Thorough peer review

- Inclusion in PubMed and all major indexing services

- Maximum visibility for your research

Submit your manuscript at www.biomedcentral.com/submit

) Biomed Central 